\title{
Retroperitoneal Cyst: An Uncommon Presentation of Filariasis
}

\author{
Senthil Ganesan, Saurabh Galodha, and Rajan Saxena \\ Department of Surgical Gastroenterology, Sanjay Gandhi Post Graduate Institute of Medical Sciences, Lucknow 226014, India \\ Correspondence should be addressed to Senthil Ganesan; jeeyes1@gmail.com
}

Received 12 September 2015; Revised 5 November 2015; Accepted 5 November 2015

Academic Editor: Christoph Schmitz

Copyright (C) 2015 Senthil Ganesan et al. This is an open access article distributed under the Creative Commons Attribution License, which permits unrestricted use, distribution, and reproduction in any medium, provided the original work is properly cited.

\begin{abstract}
Primary retroperitoneal parasitic cysts are rare. Here we report about a middle aged male patient from rural north India with a recent onset of central abdominal retroperitoneal lump, pain, and fever. After surgical resection due to diagnostic uncertainty, at histopathology, it turned out be a filarial cyst. After receiving a course of diethylcarbamazine, the patient is asymptomatic at 4 months' follow-up.
\end{abstract}

\section{Introduction}

Primary retroperitoneal parasitic cysts are uncommon $[1$, 2]. Mostly these are echinococcal cysts [3]. Retroperitoneal filarial cysts are very rare even in endemic areas like India and there are only few case reports $[4,5]$. Here we report an unusual presentation of filariasis as retroperitoneal cyst.

\section{Case Presentation}

A 35-year-old gentleman, from rural north India, presented with a progressive, central abdominal lump for one-month duration. Three weeks after the appearance of the lump, he developed intermittent high grade fever and dull aching abdominal pain. He did not have any significant past illness or comorbidities. On examination, he had a moderately tender abdominal lump predominantly in the umbilical region. Apart from nontender thickening of the right spermatic cord, there was no scrotal or testicular abnormality. The inguinal nodes were barely palpable and there was no lower limb edema.

Complete blood counts and blood chemistry were within normal limits. Blood culture was sterile. Abdominal sonography revealed a large, well defined, unilocular cyst of $20 \times$ $14 \times 23 \mathrm{~cm}$ size, located behind the right colon and terminal ileum. There were no features suggestive of an abscess. The rest of the organs were normal. Contrast enhanced CT scan confirmed the sonographic findings without adding any other information. The origin and etiology of the cyst could not be delineated (Figure 1).

Due to diagnostic uncertainty and association of symptoms like pain, the abdomen was explored revealing a large retroperitoneal cyst behind the right colon, mesocolon, and terminal ileum, stretching the organs over it. As there was well defined avascular plane around the cyst, the cyst could be excised completely without any spillage. A conglomeration of grossly dilated lymphatics was seen close to upper pole of the cyst and excised along with the cyst. The rest of the organs were normal. The cut section of the cyst revealed a thick walled cyst containing minute filamentous septations and mucoid material (Figure 2). Postoperative recovery was uneventful.

Cyst fluid analysis showed total cell count of $95 / \mathrm{cmm}$ with $60 \%$ lymphocytes and $40 \%$ neutrophils. Cyst fluid culture was sterile. Histopathology of the cyst showed numerous cystic spaces packed with microfilariae and dense mixed inflammatory infiltrate in the surrounding tissues (Figure 3). It was a retroperitoneal filarial cyst! After a course of diethylcarbamazine (100 mg t.i.d. for 3 weeks), he is asymptomatic at 4 months' follow-up.

\section{Discussion}

Primary retroperitoneal cysts are rare, often asymptomatic, and incidentally detected. They have been classified as (a) urogenital cysts, (b) mesocolic cysts, (c) cysts arising in cell inclusions, (d) traumatic cysts, (e) parasitic cysts, and 


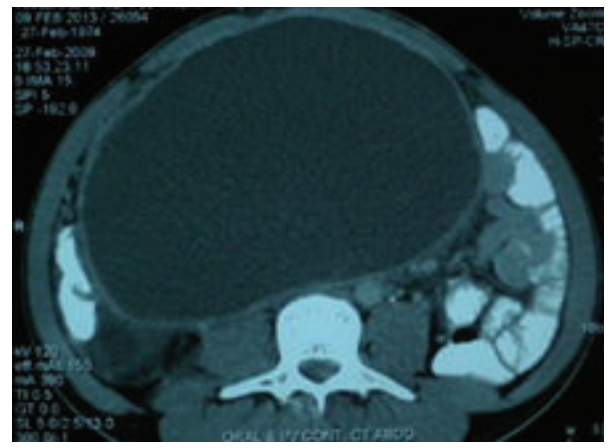

FIGURE 1: Contrast enhanced CT scan showing retroperitoneal cyst.
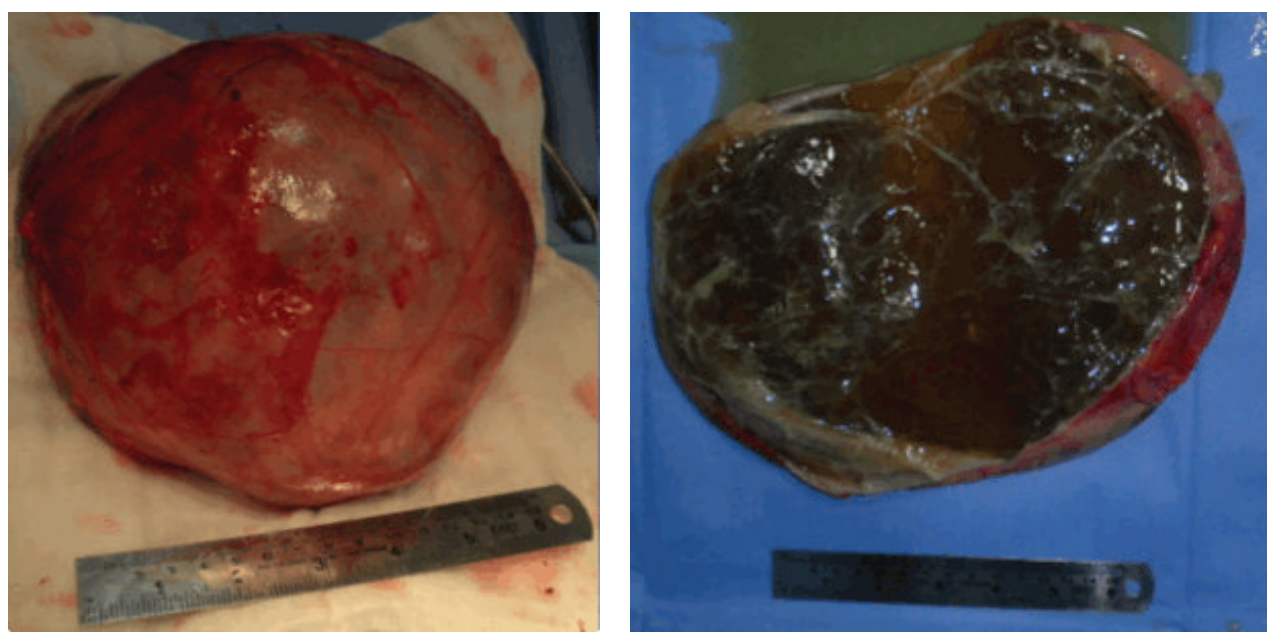

FIGURE 2: Excised retroperitoneal filarial cyst and its cut section.
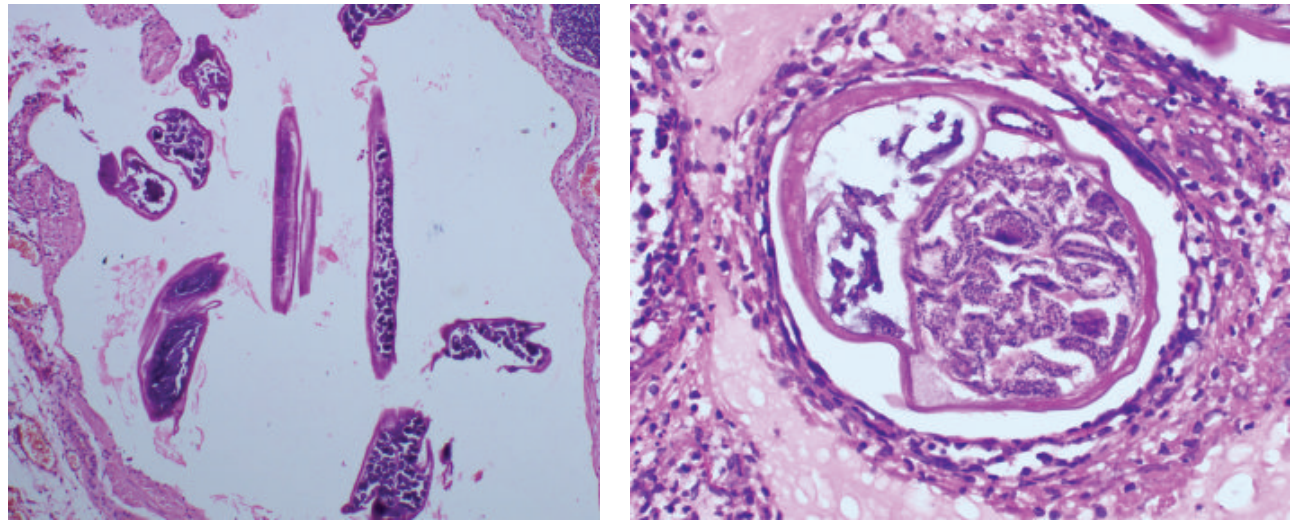

FIGURE 3: Histopathology of cyst showing microfilaria.

(f) lymphatic cysts, depending on the origin and histology $[1,2]$. Often primary retroperitoneal parasitic cysts are echinococcal cysts and Aydinli et al. reported a series of 14 cases [3].

Filariasis presenting as a retroperitoneal cyst is extremely uncommon. Reported incidence of filarial retroperitoneal cyst in hospitalized patients is $1 / 105000[6,7]$. To our knowledge, there are only four case reports of filariasis presenting as retroperitoneal cyst, abscess, or mass and most of them are from India $[4,5,8,9]$. There are also reports of filariasis presenting as abscess [8] and heterogenous mass mimicking retroperitoneal tumor [9].

Kapoor et al. reported about a patient with similar presentation except the duration of symptoms was longer (6 months) and patient also had bilateral hydrocele [4]. They established the diagnosis by fine needle aspiration 
cytology (FNAC). As it did not regress after 4 weeks of diethylcarbamazine therapy, they ultimately needed to excise the cyst. Giri et al. reported about a young male patient presenting with heterogenous mass at left iliac fossa along with left sided hydrocele and hydronephrosis of left kidney [9]. After confirming the diagnosis by FNAC, the patient was treated with antifilarial therapy which led to almost complete resolution of the cyst.

Among all 8 species of filarial worms, the most common organism encountered in India is Wuchereria bancrofti. Common presentations are asymptomatic microfilaremia, acute adenolymphangitis, hydrocele, and lymphedema [10]. Recent onset of retroperitoneal cyst, without other common manifestations of filariasis like hydrocele and lymphedema, is the peculiar presentation of our patient. We decided to perform surgery straight away without FNAC, as the patient had large symptomatic cyst with diagnostic uncertainty. If we analyze retrospectively, high grade fever with normal leucocyte count, no bacteremia on blood culture, and a subtle sign of right sided spermatic cord thickening in a patient from the background of rural north India (endemic areas) could have been the clues. Imaging rarely helps to clinch the diagnosis. Often the diagnosis is made either by FNAC or histopathology after excision like in our case. In smaller cysts, if confirmed by preoperative investigations like FNAC, one can start with medical treatment first and surgical excision may be reserved for persistent symptomatic cysts.

\section{Conclusion}

Even though filarial retroperitoneal cysts are uncommon, in endemic areas it must be included in the differential diagnosis of the retroperitoneal cysts.

\section{Consent}

Written informed consent was obtained from the patient for publication of this case report and any accompanying images.

\section{Conflict of Interests}

The authors declare that they have no competing interests.

\section{Authors' Contribution}

All authors participated in the operation or management of the patient in this case report. Senthil Ganesan, Saurabh Galodha, and Rajan Saxena drafted and revised the paper. Rajan Saxena is a chairperson of our department and supervised the writing of the paper. All authors read and approved the final paper.

\section{References}

[1] M. W. Guile, M. Fagan, A. Simopolous, and M. Ellerkman, "Retroperitoneal Cyst of Müllerian Origin: a case report and review of the literature," Journal of Pelvic Medicine and Surgery, vol. 13, no. 3, pp. 149-152, 2007.
[2] R. M. Handfield-Jones, "Retroperitoneal cysts: their pathology, diagnosis, and treatment," British Journal of Surgery, vol. 12, no. 45, pp. 119-134, 1924.

[3] B. Aydinli, G. Ozturk, K. Y. Polat et al., "Extravisceral primary hydatid cyst of the retroperitoneum," ANZ Journal of Surgery, vol. 77, no. 6, pp. 455-459, 2007.

[4] A. K. Kapoor, S. K. Puri, A. Arora, L. Upreti, and A. S. Puri, "Case report: filariasis presenting as an intra-abdominal cyst," Indian Journal of Radiology and Imaging, vol. 21, no. 1, pp. 1820, 2011.

[5] C. R. Pillay, "Retroperitoneal cyst of filarial origin," Journal of the Association of Physicians of India, vol. 9, pp. 760-762, 1961.

[6] M. Madhavan, S. K. Vanaja, K. Chandra, and D. J. Reddy, "Atypical manifestations of filariasis in Pondycherry," Indian Journal of Surgery, vol. 34, pp. 392-394, 1972.

[7] G. Chittipantulu, K. Veerabhadraiah, G. V. Ramana, and M. V. Somayajulu, "Retroperitoneal cyst of filarial origin," Journal of the Association of Physicians of India, vol. 35, no. 5, pp. 386-387, 1987.

[8] R. B. Mehta, V. Gajendran, N. Ananthakrishnan, and S. Parkash, "Retro peritoneal tumorous lesions: a clinicopathological study," Indian Journal of Surgery, vol. 43, pp. 731-742, 1981.

[9] A. Giri, A. K. Kundu, M. Chakraborty, and S. Das, "Microfilarial worms in retroperitoneal mass: a case report," Indian Journal of Urology, vol. 17, pp. 57-58, 2000.

[10] A. S. Fauci, E. Braunwald, D. L. Kasper et al., Harrison's Principles of Internal Medicine, McGraw Hill, New York, NY, USA, 17th edition, 2008. 


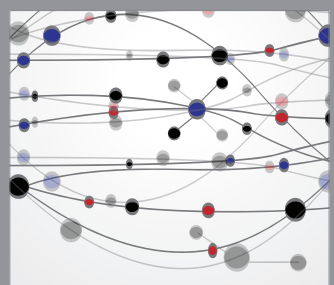

The Scientific World Journal
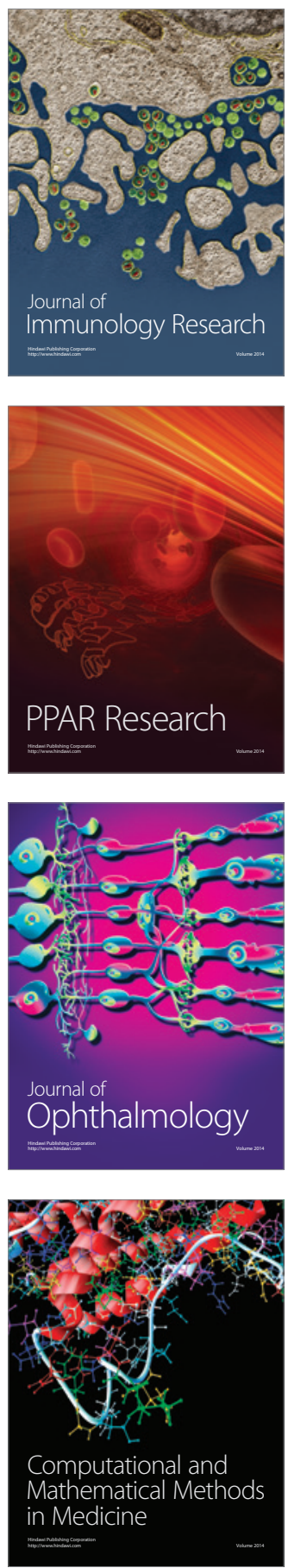

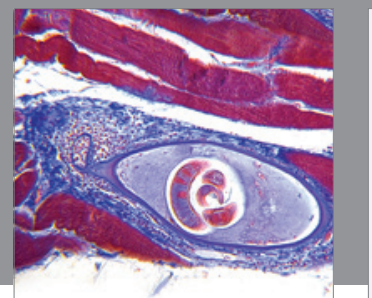

Gastroenterology

Research and Practice
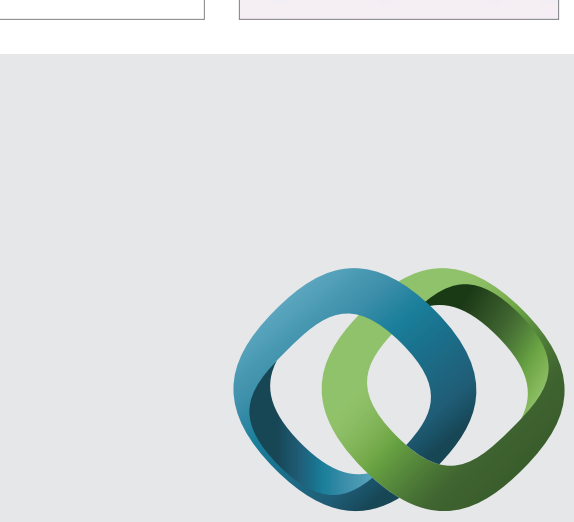

\section{Hindawi}

Submit your manuscripts at

http://www.hindawi.com
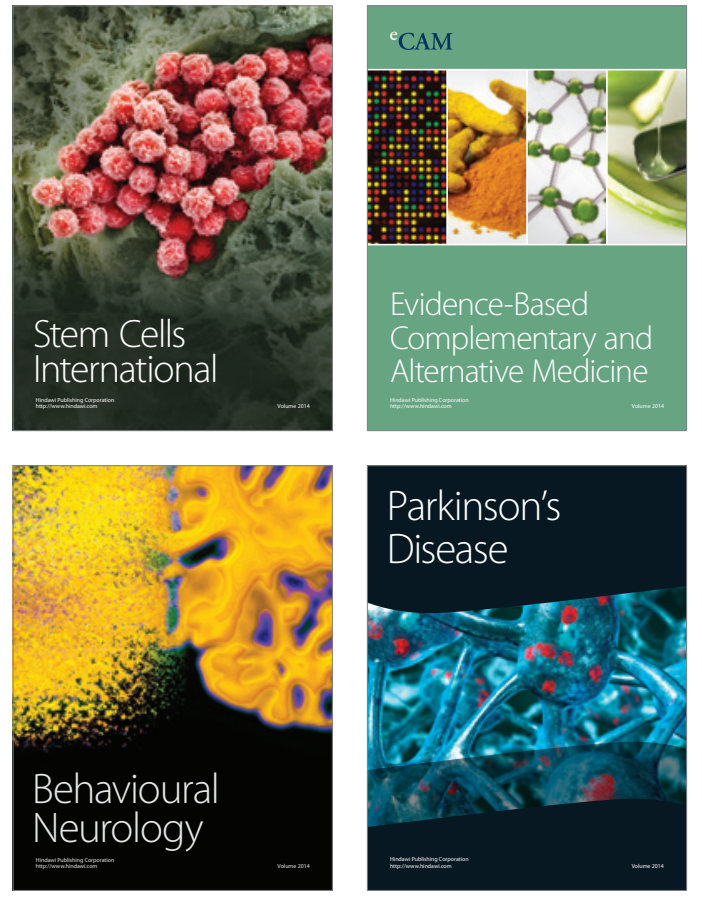
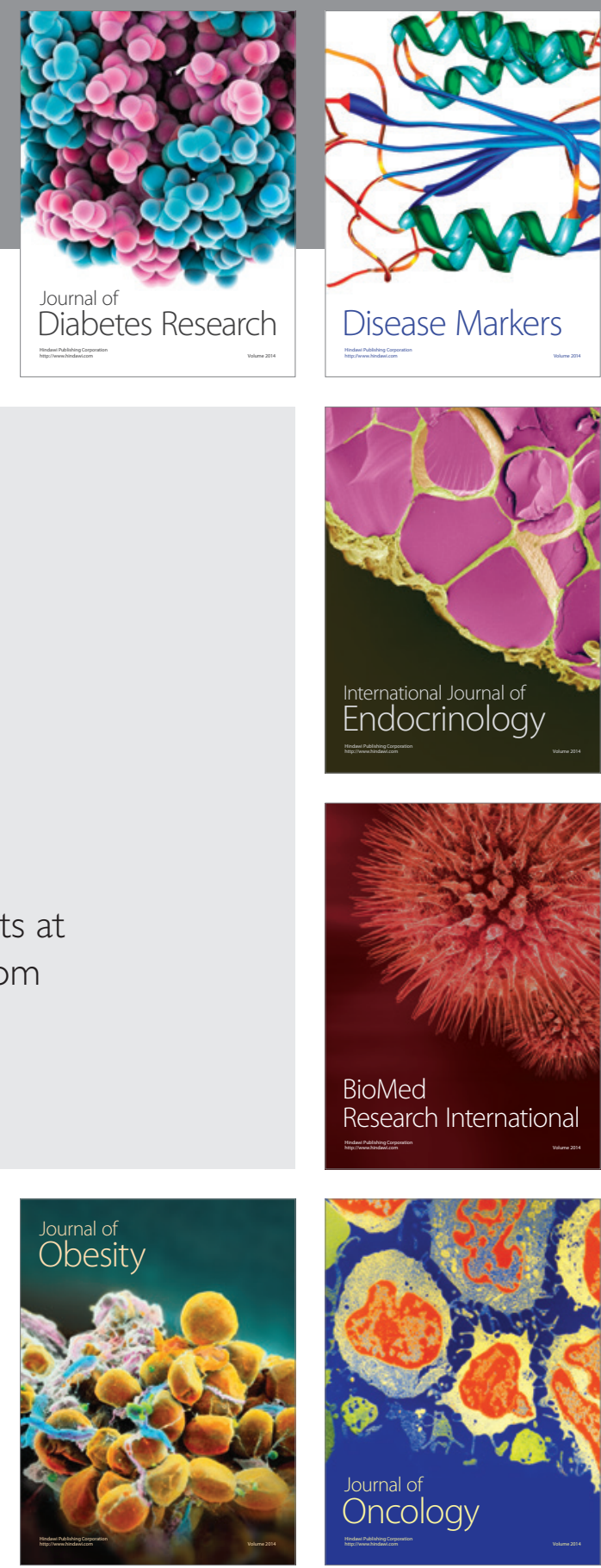

Disease Markers
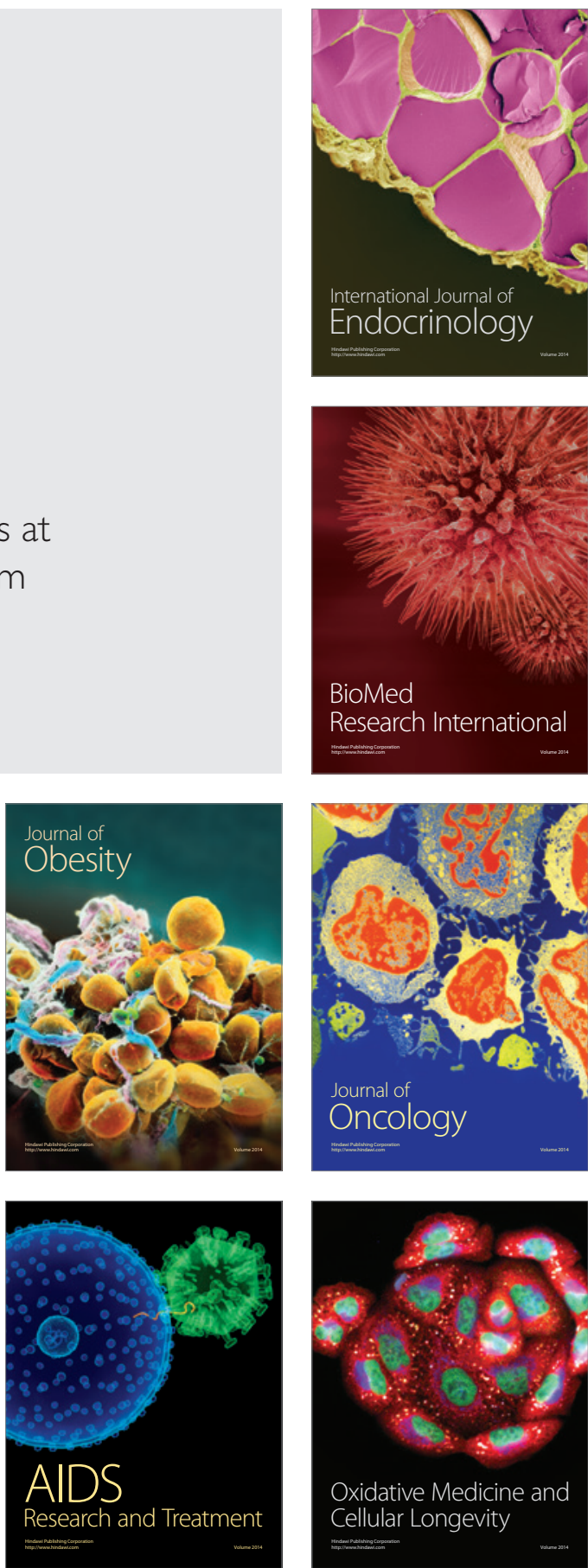